\title{
Editorial: National Conference on Nano/Bio-Technology 2019, India
}

\author{
G. B. V. S. Lakshmi ${ }^{1}$, Ajeet Kaushik ${ }^{2}$, Anil Kumar ${ }^{3}$ and Pratima R. Solanki ${ }^{1 *}$ \\ ${ }^{1}$ Special Centre for Nanoscience, Jawaharlal Nehru University, New Delhi, India, ${ }^{2}$ NanoBioTech Laboratory, Department of \\ Natural Sciences, Division of Sciences, Art, and Mathematics, Florida Polytechnic University, Lakeland, FL, United States, ${ }^{3}$ Gene \\ Regulation Laboratory, National Institute of Immunology, New Delhi, India
}

Keywords: nanobiosensor, drug deleivery, bio-imaging, nanotoxicity, nanomedicime

Editorial on the Research Topic

National Conference on Nano/Bio-Technology 2019, India

The National Conference on Nano/Bio-Technology 2019 organized jointly by the Jawaharlal Nehru University and the National Institute of Immunology, New Delhi, India during December 19 to 21,2019 . This multidisciplinary conference focused on state-of-art synergies between nanoscience and biotechnology, and their significant technological aspects. As a result, the attending scholars gained cutting edge knowledge of nano-supported technological advancements for developing nanomedicine, nano-biosensors, point of care devices, and other biomedical devices relating to health care. The key scientific findings presented in this conference were published as a special issue in Frontiers in Nanotechnology under the title "National Conference on Nano/Bio-Technology 2019, India" (https://www.frontiersin.org/

\section{OPEN ACCESS}

Edited and reviewed by:

Themis Prodromakism,

University of Southampton, United Kingdom

${ }^{*}$ Correspondence:

Pratima R. Solanki

partima@mail.jnu.ac.in

Specialty section:

This article was submitted to

Biomedical Nanotechnology,

a section of the journal

Frontiers in Frontiers in

Nanotechnology

Received: 15 July 2021

Accepted: 02 August 2021

Published: 07 September 2021

Citation:

Lakshmi GBVS, Kaushik A, Kumar A

and Solanki PR (2021) Editorial:

National Conference on Nano/Bio-

Technology 2019, India.

Front. Nanotechnol. 3:742043.

doi: 10.3389/fnano.2021.742043 research-topics/15270/national-conference-on-nanobio-technology-2019-india). In this special issue, the editors selected nanomaterials-based articles that deal with applications in biosensors, drug delivery, nanotoxicity, etc. These articles were contributed a variety of reputed Indian universities, national laboratories, and research institutions with the aim to bring together scholars of various disciplines, including nanomaterials, enabled biotechnology, nano-biosensors, bio-imaging, drug delivery, diagnostic devices, nano-toxicity, and microbiology.

Wide varieties of nano/bio-technology related topics were covered in this special issue. The electrochemical sensors based on different nanomaterials for various analytes were reported. A new electrochemical sensor was reported, based on $\beta$-tricalcium phosphate $\left(\mathrm{Ca}_{3}\left(\mathrm{PO}_{4}\right)_{2}\right)$ nanoparticles modified by a glassy carbon electrode for the selective non-enzymatic determination of methyl parathion and mercury (II) ions independently. This sensor has high selectivity towards MP and $\mathrm{Hg}$ in the presence of major interfering compounds such as 3-nitrophenol, 4-nitrophenol, 4aminophenol, catechol, hydroquinone and heavy metals such as lead, cadmium and arsenic. Applicability of the fabricated sensor for detection of MP and Hg (II) ions has been tested in tap water by the standard addition method. An electrochemical sensor for the detection of cholesterol based on thin films of nafion (polymer) and hematite or $\alpha-\mathrm{Fe}_{2} \mathrm{O}_{3}$ (nanoparticles) nanocomposite was fabricated on indium tin oxide (ITO) coated glass substrates.

The synergy between the antifungal drugs and the $\mathrm{ZnO}$ quantum dots was studied, and it was found that the incorporation of quantum dots can decrease dose size in the treatment of fungal infections leading to reduces toxicity, and their multitargeted action could limit evolution of fungal drug resistance. Gelatin nanoparticles were used for therapeutic applications: the Etoposide loaded gelatin nanoparticles (EGNPs) were tested for in-vivo therapeutic potential by studying pharmacokinetics, biodistribution and tumor regression in Ehrlich Ascites Carcinoma (EAC), 
using a mice model. High biocompatibility and bio-efficacy of EGNPs proved their therapeutic potential in cancer treatment.

Two different nanomaterials based optical sensors were reported for the detection of toxins and antibiotics. Manganese oxide nanoparticles $\left(\mathrm{MnO}_{2} \mathrm{nps}\right)$ were explored to study the interaction with aflatoxin (AFB1) and ochratoxin (OchA) using UV-visible spectroscopy. This bio-active free direct sensing approach of AFB1 and OchA sensing was reported as a potential analytical tool to estimate food quality rapidly and in an affordable manner at the point of use. The L-Cysteine capped magnesium sulfide quantum dots (L-Cyst-MgS QDs) were used to detect Levofloxacin antibiotic via the photoinduced electron transfer mechanism. The fluorescence of the QDs was used as the sensing platform for the selective detection of Levofloxacin antibiotic.

The oxidative stress and immunotoxicity imposed by Zinc selenium/Zinc sulphide quantum dots (ZnSe/ZnS QDs) was studied and reported. The higher concentrations of the $\mathrm{ZnSe} /$ ZnS QDs causes cell death due to reactive oxygen species production. The study evidenced that $\mathrm{ZnSe} / \mathrm{ZnS}$ QDs is not capable of eliciting any serious damages to liver and brain tissues, which in turn substantiates its applicability in biomedical applications. The cerium doped tin oxide nanoparticles (Ce doped $\mathrm{SnO}_{2}$ nps) were studied for their antimicrobial activity and photocatalytic activity. The Ce doped $\mathrm{SnO}_{2}$ nps prevented the E.coli bacterial growth, and were successfully used for the photocatalytic degradation of Malachite Green dye under UV light irradiation.

A mathematical modelling of blood flow with the suspension of nanoparticles through a tapered artery with a blood clot was reported. Also, a small review was given on the applications of 3D-printed microfluidics in biomedical applications. The paper reviewed the emerging diagnostic technologies using 3D printing as a method for integrating living cells or biomaterials into $3 \mathrm{D}$ printing. It is important to have standards and regulations in the device development used in health care sector. The National Physical Laboratory (NPL), India, is playing a significant role in this aspect. A brief the summary on the significant achievements and role of NPL in supporting the Indian medical device regulations was presented.

Overall, the articles published in this special issue projected high-performance smart nano-systems for various biomedical applications focused on the detection, treatment, and monitoring of a targeted marker. At the same time, scholars also raised the concern of limited resources and lacking translational and sustainable efforts to introduce nanoscience for industrial and clinical application. The editor also supports this view, and believes that in future more efforts will be made to divert fundamental research of nano-biotechnology towards translational efforts. In this process, the Frontiers in Nanotechnology journal will always be one of the best platforms to publish such top ranked research to fill the gap between education and applied technology.

\section{AUTHOR CONTRIBUTIONS}

All the authors have contributed to prepare the special issue of the conference and gave significant inputs for writing the editorial card.

\section{ACKNOWLEDGMENTS}

We acknowledge the Frontiers in Nanotechnology journal staff for providing the opportunity and guidance to publish the special issue in your esteemed journal. The authors are also thankful to the Science and Engineering Research Board, the Department of Science and Technology and the Department of Biotechnology Indo-Russia (DBT/IC-2/ Indo-Russia/2017-19/02) project, Government of India for financial support for National Conference on Nano/BioTechnology 2019, India.

Conflict of Interest: The authors declare that the research was conducted in the absence of any commercial or financial relationships that could be construed as a potential conflict of interest.

Publisher's Note: All claims expressed in this article are solely those of the authors and do not necessarily represent those of their affiliated organizations, or those of the publisher, the editors and the reviewers. Any product that may be evaluated in this article, or claim that may be made by its manufacturer, is not guaranteed or endorsed by the publisher.

Copyright $\odot 2021$ Lakshmi, Kaushik, Kumar and Solanki. This is an open-access article distributed under the terms of the Creative Commons Attribution License (CC $B Y)$. The use, distribution or reproduction in other forums is permitted, provided the original author(s) and the copyright owner(s) are credited and that the original publication in this journal is cited, in accordance with accepted academic practice. No use, distribution or reproduction is permitted which does not comply with these terms. 